\title{
La construction à verbes de nomination : la voilà parachutée construction!
}

\author{
Justine Métairy ${ }^{1}$ \\ Ghent University, Belgium
}

\begin{abstract}
Résumé. Dans cet article, nous nous proposons de dresser l'inventaire des verbes qui apparaissent dans l'une des rares constructions dites « résultatives » du Français, i.e. la Construction à Verbes de Nomination $(=\mathrm{CVN})$ (ex. Napoléon est sacré empereur des Français). Sur la base des résultats de notre recherche, nous démontrons que la CVN est bel et bien une construction (dans le sens où on l'entend dans la Grammaire des Constructions (cf. Goldberg 1995)), qui s'avère, par ailleurs, très productive : en effet, la CVN est capable d'attirer un grand nombre de verbes appartenant à des classes sémantiques diverses et - étant dotée d'un pouvoir coercitif important - de faire fi des spécifications lexicales de ces derniers.
\end{abstract}

\begin{abstract}
The nomination verb construction: now catapulted to (the rank of) construction! In this article, we take up the challenge to list the verbs that occur in one of the rare "resultative" constructions available in French, i.e. the Nomination Verb Construction ( $=$ NVC) (e.g. Napoléon est sacré empereur des Français 'Napoléon is consecrated emperor of the French'). Based on the results of our research, we show that the $\mathrm{NVC}$ is a construction (in the sense of Construction Grammar (cf. Goldberg 1995)), which turns out to be quite productive: indeed, it appears that a great number of verbs - which belong to various semantic classes - are accepted in the NVC, sometimes independently of their specific lexical restrictions.
\end{abstract}

\section{Introduction : la Construction à Verbes de Nomination}

La Construction à Verbes de Nomination $(=\mathrm{CVN})$ est une construction dans laquelle le référent dénoté par le complément d'objet direct du verbe est soumis à un changement de statut au terme du procès de nomination dénoté par le verbe. Tout au long de cet article, nous utiliserons le terme de «nomination » pour désigner tout acte par lequel une entité (animée ou inanimée) se voit attribuer un statut officiel (ex. un titre, un emploi, une charge etc.). Comme illustré dans les exemples suivants, il s'agit généralement de procédures officielles comme, par exemple, un couronnement (cf. (1)), une élection (cf. (2)), une proclamation (cf. 3)) ou encore une promotion (cf. (4)) :

(1) Le 2 décembre 1804, Napoléon couronne Joséphine impératrice de France. ${ }^{\mathrm{i}}$

(2) Argentine : Alberto Fernandez élu président dès le premier tour de l'élection.

(3) Le 5 juillet 1962, le pays est proclamé indépendant. 
AXWAY : le directeur financier est promu directeur général.

La CVN fait partie de la famille des Constructions Résultatives Analytiques ${ }^{\text {ii }}$ (=CRA) (ex. Marie a peint la porte en rouge, Jean a coupé les navets en dés) qui sont réputées pour être très productives dans les langues germaniques (cf. Carrier \& Randall 1992, Goldberg 1995, Rappaport Hovav \& Levin 1998, Boas 2003, Goldberg \& Jackendoff 2004, entre autres) mais plutôt rares dans les langues romanes, dont le français iii (cf. Riegel 1996, Legendre 1997, Muller 2000, Dagnac 2009). En effet, en plus de leur ressemblance formelle - la CVN et la CRA étant deux constructions à attribut de l'objet (cf. Guimier 1999, Tobback 2005) - ces dernières partagent la même interprétation sémantique : en effet, dans les deux cas, le procès décrit par le verbe est à l'origine du changement d'état (ou du statut en ce qui concerne les CVNs) auquel est soumis l'objet du verbe. Bien sûr, les CVNs et les CRAs divergent sur quelques aspects : alors que le prédicat second résultatif ( $=$ PSR) est très souvent instancié par un nom, et plus précisément, par un nom nu ${ }^{\mathrm{iv}}$ dans les CVNs (ex. impératrice de France en (1), président en (2) et directeur général en (4)) (cf. Métairy \& al. (2020)), ce dernier est généralement exprimé par un groupe prépositionnel (ex. en rouge, en dés) ou par un adjectif ${ }^{\mathrm{v}}$ dans les CRAs.

Dans la littérature, la classe des verbes qui entrent dans la CVN (qui se sont vu attribuer plusieurs étiquettes, ex. « verbes factitifs d'état » dans Willems (1981), « verbes performatifs » dans Riegel (1991), " verbes causatifs-performatifs » dans Riegel (2001) ou encore « verbes causatifs » dans Pierrard (2001)) vi se limitent à une poignée de membres. Cependant, l'emploi de certains verbes, ex. bombarder en (5), dont le sens est « forcé » ou « coercé » (cf. Lauwers \& Willems 2011) par la CVN de sorte à ce qu'ils soient également interprétés comme verbes de nomination, suggère que la construction est productive ${ }^{\text {vii }}$.

(5) Dali Benssalah, le jeune Français bombardé tête d'affiche dans le nouveau «James Bond».

\section{Objectifs de notre étude}

Tout d'abord, cette étude vise à dresser l'inventaire des verbes qui apparaissent dans la CVN en français. Comme nous l'avons mentionné plus haut, le fait que la CVN puisse attirer des verbes qui ne décrivent pas a priori un procès de nomination (cf. bombarder en (5)) laisse à penser que d'autres verbes puissent être attestés dans cette construction. Nous démontrerons, par la suite, que postuler l'existence d'une CVN dans le sens de la Grammaire des Constructions (cf. Goldberg 1995) permet d'expliquer les différents phénomènes de coercition que nous avons observés au cours de cette recherche. En retour, ces effets de coercition donnent du crédit à l'hypothèse selon laquelle la CVN est bel et bien une construction (supra-lexicale), dotée d'un sémantisme sui generis, capable de modifier les spécifications lexicales des items qui y entrent. 


\section{Méthodologie : délimitation de la recherche et collecte des données}

Avant de présenter les résultats de notre recherche, il convient de dire quelques mots sur la méthodologie que nous avons utilisée pour amasser les verbes qui entrent dans la construction. À noter que la définition de la CVN donnée dans la Section 1 nous a servi à délimiter cette recherche. Plusieurs types de constructions verbales ont été ainsi écartées :

(i) Les constructions à verbes de dénomination (ex. appeler, nommer, dénommer, surnommer) :

Même si les verbes de dénomination sont très souvent cités dans la littérature comme faisant partie de la même classe que les verbes de nomination, nous avons décidé de les exclure, et ceci pour les raisons suivantes : tout d'abord, d'un point de vue sémantique, le prédicat second - bien qu'il soit aussi résultatif - ne dénote pas un statut officiel, mais un nom propre. Les CVNs et les constructions à verbes de dénomination divergent également sur le plan syntaxique : par exemple, contrairement à ce qu'on observe dans les CVNs, l'omission du PSR est impossible dans les constructions à verbes de dénomination (ex. On l'a promu (directeur) vs. On l'a surnommé *("le grand »)). Enfin, alors que dans les CVNs, le PSR peut être marqué syntaxiquement par un « marqueur prédicatif » (cf. Lauwers \& Tobback 2018 et Métairy \& al. (2020)), ceci n'est pas possible avec le PSR des constructions à verbes de dénomination (ex. On l'a élu comme président vs. *On l'a surnommé comme «le grand»).

(ii) Les constructions dites « dépictives » :

Dans certains cas, le prédicat second peut renvoyer à un état (ou à un statut comme en (6)) qui, au lieu d'être causé par le procès dénoté par le verbe, est concomitant à celuici - on parle alors de prédicats « dépictifs » (cf. Haliday 1967). Ne décrivant pas des situations qui mènent à un changement de statut, les constructions dépictives, telles que (6), ont été exclues de notre base de données.

Il y a quelques mois, presque personne ne l'imaginait président de la République.

Cependant, il est important de mentionner le fait que certains verbes ont été attestés dans la CVN, alors que ces derniers sont le plus souvent associés à une interprétation dépictive. C'est le cas, par exemple, de considérer dans l'exemple (7) :

(7) Considéré monument historique par la législation des Monuments Historiques établie en 1914, il est inscrit au patrimoine mondial de l'Unesco en 1985. 
Par ailleurs, notez que l'emploi du nom nu monument historique en position de PRS avec le verbe considérer est très certainement légitimé par la CVN. Autrement le PRS apparaitrait avec un article et serait également précédé d'un marqueur prédicatif (ex. je le considère comme un moins que rien/le diable en personne).

Passons à présent au protocole d'extraction des données. Dans un premier temps, nous avons dressé une première liste de verbes de nomination sur la base des verbes cités dans la littérature comme apparaissant dans la CVN (cf. Section 1). Nous y avons ajouté, par la suite, des synonymes et des antonymes (tirés de dictionnaires de synonymes en ligne, ex. Crisco). Nous nous sommes assurée que ces derniers entrent effectivement dans la CVN, et ceci par différents moyens : (i) en utilisant des dictionnaires unilingues (cf. le Trésor de la langue française informatisé (TLFi) et le Larousse) ou (ii) en cherchant la combinaison « verbe + PSR (ex. président, roi) » sur le moteur de recherche Google dans les cas où il n'y est pas mentionné que le verbe puisse s'employer ainsi. Nous avons également collecté des verbes de nomination par l'intermédiaire d'un corpus web étiqueté (Le French Ten Ten Corpus de Sketch Engine) via le PSR - méthode également appliquée chez Boas (2003). Concrètement, nous avons dressé la liste des 40 PSRs qui apparaissent le plus fréquemment avec un verbe de nomination relativement fréquent, i.e. nommer, et nous avons ensuite récupéré tous les verbes qui se combinent avec ces PSRs et qui ont, bien sûr, un sens résultatif. Enfin, pour terminer, nous avons fait contrôler l'acceptabilité de ces verbes dans la CVN par un locuteur natif.

\section{Résultats}

\subsection{Présentation générale des données}

Au total, nous avons collecté 140 verbes qui apparaissent dans la CVN en français. Comme illustré dans le Tableau 1, ces verbes ont été répartis dans 7 classes sémantiques différentes en fonction du type de procès de nomination qu'ils décrivent (voir le Tableau 2 en Annexe pour avoir un aperçu complet des données) :

\begin{tabular}{|l|c|}
\hline Les classes sémantique & Nombre de verbes \\
\hline Les verbes du type couronner & 40 \\
\hline Les verbes du type promouvoir & 21 \\
\hline Les verbes du type désigner & 15 \\
\hline Les verbes du type déclarer & 11 \\
\hline Les verbes du type confirmer & 14 \\
\hline Les verbes du type diagnostiquer & 19 \\
\hline Les verbes du type classer & 16 \\
\hline Total & $140^{\text {viii }}$ \\
\hline
\end{tabular}

Tableau 1. Nombre de verbes de nomination pour chaque classe sémantique 


\subsection{Les différentes classes sémantiques}

\subsubsection{Les verbes du type couronner}

Comme on peut le constater sur le Tableau 1, les verbes du type couronner constitue la plus grande classe sémantique de notre base de données (i.e. 40 verbes au total). Ces derniers peuvent être répartis en trois sous-classes sémantiques. La première contient des verbes qui décrivent des cérémonies et des rituels bien spécifiques (ex. couronnement, adoubement, investiture) :

(8) Le 2 décembre 1804, Napoléon couronne Joséphine impératrice de France.

(9) À Bonaguil, Terkal le Normand a été adoubé chevalier.

(10) Elle fut canonisée Sainte par le Pape Jean-Paul II en 1982 [...].

(11) L'ex-comédien et humoriste Volodymyr Zelensky a été investi président d'Ukraine lundi.

(12) Cette dernière lui réclame 550 euros au motif qu'il a été baptisé catholique à sa naissance.

(13) Rugby : naturalisé francais, Raka (Clermont) devient sélectionnable.

Notez que ces verbes sont parfois associés à un sens factitif ou causatif même lorsqu'ils n'apparaissent pas dans la CVN. Ainsi, 5 des 6 verbes illustrés dans les exemples (8)-(13) acceptent tous la paraphrase « faire être + PSR » même lorsqu'ils ne se combinent pas avec un PSR (cf. (14)). En revanche, lorsque ces verbes sont utilisés dans la CVN, le PSR vient alors spécifier ou expliciter le statut qui est déjà compris dans la sémantique du verbe.

(14) adouber $\Rightarrow>$ 'faire être chevalier', couronner $\Rightarrow>$ 'faire être roi ou reine', baptiser $=>$ 'faire être chrétien', naturaliser $=>$ 'faire être citoyen (d'un pays donné)', canoniser $=>$ 'faire être saint'

La deuxième sous-classe regroupe des verbes tels que instaurer (cf. (15)), instituer (cf. (16)) et introduire (cf. (17)) (cf. Tableau 2). Contrairement aux verbes de la sousclasse précédente, ces derniers ont un sémantisme assez général. Par conséquent, il est impossible d'en déduire le genre de statut dénoté par le PSR.

(15) Au lendemain de la mort de l'évêque, le Protestantisme est instauré comme religion officielle.

(16) Il a institué Jean son unique héritier. (cf. Riegel 1991)

(17) Récemment, Benoît Cerexhe a été introduit président du Belgium Beer Club $[\ldots]$

La troisième sous-classe comporte des verbes qui décrivent des cérémonies durant lesquelles quelqu'un reçoit un prix, une récompense ou encore une distinction, comme 
par exemple avec les verbes décorer, médailler et oscariser (cf. Tableau 2). Lorsque ces verbes se combinent avec un PSR, celui-ci spécifie la distinction en question :

(18) Le père de Cyril Hanouna décoré chevalier de la Légion d'honneur.

(19) Un événement lors duquel Lohann Adjutor a été médaillé champion de Bretagne et Thomas Huet vice-champion de Bretagne, tous deux en catégorie minime $(-57 \mathrm{~kg})$.

(20) Rami Malek, oscarisé meilleur acteur pour son rôle de Freddie Mercury, pourrait incarner le méchant dans le prochain James Bond.

\subsubsection{Les verbes du type promouvoir}

Les verbes du type promouvoir constituent la deuxième classe la plus importante de notre base de données (cf. Tableau 1). Ces verbes décrivent des situations durant lesquelles quelqu'un se voit attribuer un rang supérieur (ou inférieur) au sein d'une organisation. Dans de nombreux cas, ces verbes véhiculent l'idée d'un déplacement métaphorique, qui peut être perçu de différentes façons : de manière neutre (ex. se hisser (cf. (21)) et rétrograder (cf. (22)) - en plus de promouvoir (cf. (4))), de manière soudaine (ex. catapulter (cf. (23)) et parachuter (cf. (24)) - en plus de bombarder (cf. (5)) ou encore de manière péjorative (ex. balancer (cf. (25)) et pousser (cf. (26))) :

(21) Ainsi, en 1999, il s'est hissé chef des cuisines de L'Amphitryon, à Colomiers, près de Toulouse.

(22) Rétrogradé remplaçant par Marc Lièvremont depuis le match contre la Nouvelle-Zélande, l'ouvreur des Bleus François Trinh-Duc promettait qu'il faudrait compter sur lui jusqu'au bout.

(23) Catapulté ministre wallon des pouvoirs locaux il y a 3 mois, en pleine crise Publifin, il n'a pas abandonné toute ambition pour sa ville.

(24) Suite au départ d'Alain Juppé pour causes judiciaires, Nicolas Sarkozy est parachuté président de l'UMP avec $85,1 \%$ des voix.

(25) J'ai été balancé président par mes amis et j'ai assumé le poste jusqu'à il y a peu.

(26) J'ai été poussé chef d'équipe (d'une équipe de bac+5) avec un bac+2 et 3 mois d'expérience, à la fois par le chef de projet et par tous les membres de l'équipe, qui ne voyaient personne d'autre pour faire ce boulot.

Notez que ces verbes n'ont jamais été cités dans la littérature comme verbes de nomination. Sans doute parce que, contrairement à d'autres (ex. couronner, adouber, baptiser etc. (cf. Section 4.2.1), ces verbes ne peuvent être interprétés comme dénotant un procès de nomination que lorsqu'ils apparaissent dans la CVN, i.e. lorsqu'ils se combinent avec un PSR. Nous reviendrons sur ce point plus en détail dans la Section 5 . 


\subsubsection{Les verbes du type désigner}

Les verbes du type désigner réfèrent à un acte de désignation officiel (ex. élection, nomination, affectation etc.). Certains d'entre eux, comme par exemple désigner (cf. (27)), nommer (cf. (28)) mais aussi élire (cf. (3)), ont été régulièrement mentionnés dans la littérature portant sur les verbes de nomination :

(27) Municipales 2020 : Antoine Maurice désigné tête de liste d'Archipel Citoyen.

(28) Achim Beierlorzer a été nommé entraîneur de Mayence, a annoncé le club allemand lundi.

D'autres verbes - moins connus - peuvent également être ajoutés à cette classe. C'est le cas de coopter (cf. (29)), plébisciter (cf. (30)), reconduire (cf. (31)), sélectionner (cf. (32)) :

(29) Au bout de quelque temps, il sera coopté comme membre, ou remercié.

(30) Ali Haddad plébiscité président du FCE à 100\% des voix.

(31) Le Gendre reconduit président des députés LaREM dès le premier tour d'un vote interne.

(32) Ils ont sélectionné comme finaliste un auteur qui ne figurait pas dans leur première liste.

Remarquez que, tout en étant sémantiquement très proches du verbe élire, les verbes coopter et plébisciter s'en distinguent par certaines nuances : lorsque quelqu'un est coopté - comme c'est le cas en (29) - cela signifie qu'il a été désigné ou élu pour rejoindre une organisation (ex. la CAF) par les membres qui la composent. En revanche, lorsque quelqu'un est plébiscité, comme en (20), cela signifie qu'il a été élu par plébiscite, c'est-à-dire, de manière quasi unanime.

Enfin, l'emploi de certains verbes, tels que affecter (cf. (33)) et muter (cf. (34)) dans la $\mathrm{CVN}$, s'avère particulièrement intéressant : en effet, bien que ces verbes sélectionnent généralement un syntagme prépositionnel (ex. affecter/muter quelqu'un à la direction/au poste de directeur), ces derniers peuvent tout aussi bien apparaître dans la CVN et ainsi se combiner à un PSR nu :

(33) Le colonel Yves Métayer sera désormais affecté chef de la division opérations à l'état-major de la zone de Défense ouest à Rennes.

(34) Après le départ, en mai, de Patrick Gras, muté directeur adjoint du CHU de La Réunion, c'est Pascal Bénard, directeur de l'Établissement Public de Santé Mentale de Quimper qui a été nommé à la direction de l'EPSM de Saint-Avé. 


\subsubsection{Les verbes du type déclarer}

Les verbes du type déclarer décrivent un acte de communication, qui peut être aussi bien verbale (ex. déclarer (cf. (35)) et décréter (cf. (36)) - en plus de proclamer (cf. (3)) que non verbale (ex. acclamer (cf. (37)), ovationner (cf. (38)) et saluer (cf. (39)) (cf. Tableau 2). Ces verbes ont été très souvent cités dans la littérature comme pouvant être accompagnés d'un PSR - à l'exception peut-être du verbe ovationner, qui semble fonctionner sur le même modèle qu'acclamer.

(35) Après 35 ans en prison, un homme est déclaré innocent.

(36) C'est seulement quand le nord de la Syrie sera décrété zone d'exclusion aérienne que les YPG pourront défendre le pays sans entraves.

(37) Et comme si c'était pas assez, monsieur a été acclamé Roi du Nord, tout ça grâce à la badass de 5 ans.

(38) L'association "Les Amis du Cheval Marin" souhaitait lui rendre un hommage à sa façon et elle l'a plébiscité et ovationné_Président d'Honneur" de notre association.

(39) Aussi, à la première nouvelle de l'avènement de Vespasien, salué empereur par les légions d'Orient, ils l'acclamèrent et se déclarèrent nettement contre son compétiteur, entraînant avec eux toutes les troupes de Mésie.

Bien que ces verbes soient inclus dans la même classe sémantique, nous verrons en Section 5 qu'ils disposent de propriétés syntaxiques différentes : par exemple, contrairement aux verbes du type acclamer (i.e. ceux qui désignent un acte de communication non verbale), les verbes comme déclarer, décréter peuvent sélectionner une complétive ${ }^{\text {ix }}$. En revanche, ces derniers ne peuvent sélectionner un certain type d'objets directs seulement lorsque le PSR est également présent dans la phrase - or cette restriction ne semblent pas s'appliquer aux verbes du type acclamer.

\subsubsection{Les verbes du type confirmer}

Les verbes de type confirmer renvoient à un acte d'approbation ou de certification officiel pouvant légitimer un changement de statut. On peut mentionner à titre d'exemple les verbes agréer, authentifier, certifier, confirmer et habiliter, qui sont illustrés dans les exemples suivants :

(40) Un proche de Ouattara agréé comme ambassadeur ivoirien en France.

(41) Issu des réserves du Musée d'art et d'histoire, le tableau Vue du Lac Léman, datant de 1876, est authentifié comme toile inédite du célèbre peintre.

(42) Le chocolat Toblerone est désormais certifié Halal.

(43) Gérard Linard confirmé président fédéral de l'Union belge de football jusqu'en 2019

(44) Dans ce cas, au moins un des secouristes doit être habilité opérateur DSA consécutivement à une formation de 8 heures sur l'utilisation du DSA. 


\subsubsection{Les verbes du type diagnostiquer}

Les verbes de type diagnostiquer décrivent des procès durant lesquels une personne est soumis à un examen, une évaluation ou encore à une enquête dont le statut officiel qui en résulte est spécifié par un PSR lorsque ces derniers apparaissent dans la CVN. On trouve dans cette classe sémantique des verbes comme juger, diagnostiquer, contrôler ou encore reconnaître :

(45) En 1998, devant un jury exclusivement blanc l'homme avait été jugé coupable du meurtre d'une jeune fille blanche de 19 ans, Stacey Stites.

(46) Depuis, il a été diagnostiqué schizophrène et suit un traitement.

(47) Lot-et-Garonne : un conducteur contrôlé positif après une sortie de route au petit matin.

(48) Problème : il a été reconnu déficient mental, et sa condamnation crée un début de polémique.

Il est important de mentionner que certains verbes, ex. diagnostiquer et contrôler, peuvent parfois permuter avec d'autres verbes qui sont sémantiquement très proches, ex. déceler/détecter et vérifier respectivement, mais dont l'usage au sein de la CVN peut sembler quelque peu suspect à l'oreille d'un locuteur natif. Ces derniers semblent alors être acceptés dans la CVN sous prétexte qu'ils appartiennent au même jargon. Ainsi, les verbes diagnostiquer, déceler et détecter peuvent se substituer les uns aux autres dans certaines expressions sans que cela en change le sens (ex. diagnostiquer/déceler/détecter une maladie (ex. un cancer)). Ceci n'est pas le cas lorsque le SN postverbal est animé (ex. diagnostiquer/*déceler/*détecté un patient). Pourtant, ces restrictions de sélection ne semblent pas empêcher leur emploi dans la CVN, comme illustré dans les exemples (49) et (50) :

(49) Décelé autiste Asperger en 2004, Maxime a néanmoins suivi une scolarité traditionnelle à Bordes [...].

(50) Depuis que Thierry a été détecté séropositif en 2001, lui et son épouse se protègent systématiquement lors de leurs rapports sexuels.

Idem pour contrôler et vérifier. Étant des synonymes, ces deux verbes s'avèrent être interchangeables dans de nombreux contextes (ex. contrôler/vérifier les papiers $d u$ véhicule). Cependant, comme dans le cas précédent, le verbe contrôler peut sélectionner avec un $\mathrm{SN}$ animé, mais pas vérifier (ex. contrôler/ ??vérifier le conducteur).

(51) Vérifié_positif à l'éthylotest, il est prié de se soumettre désormais à l'éthylomètre.

\subsubsection{Les verbes du type classer}

Passons maintenant à la présentation de notre dernière classe sémantique, i.e. les verbes du type classer. Lorsque ces verbes sont utilisés dans la CVN, ils renvoient à 
des situations durant lesquelles quelqu'un (ou quelque chose) acquiert un statut en étant officiellement reconnu comme appartenant à une certaine catégorie. Cette classe sémantique inclut - entre autres - les verbes classer, estampiller, inscrire et tamponner:

(52) Le diesel officiellement classé cancérogène par l'OMS

(53) Il est devenu designer de mobilier, estampillé "spécialiste du beau".

(54) Le prieuré de Grosbois, fondé au XIIe siècle, est inscrit monument historique depuis 1929.

(55) Le jour venu - on peut prendre tous les paris -, le pays n'acceptera pas les efforts qui lui seront demandés par un nouveau chef de gouvernement, tamponné conforme par le FMI.

Notez que, dans certains cas, la CVN apparaît comme une version « condensée » d'une autre construction - peut-être plus " conventionnelle ». Ainsi, au lieu de sélectionner un nom nu comme dans les exemples (52) et (54), les verbes classer et inscrire dans les exemples (56) et (57) sélectionnent un syntagme prépositionnel qui contient ce nom :

(56) Le diesel officiellement classé dans la catégorie des substances cancérogènes par l'OMS

(57) Le prieuré de Grosbois, fondé au XIIe siècle, est inscrit sur/à la liste des monuments historiques depuis 1929.

Nous reviendrons également sur ce point dans la section 5.

Récapitulons à présent ce que nous avons observé dans cette section. On constate, tout d'abord, qu'un certain nombre de verbes peuvent apparaître dans la CVN alors même que ces derniers ne sont pas des verbes de nomination per se, c'est-à-dire qu'ils ne sont généralement pas interprétés comme décrivant un procès qui peut aboutir à un changement de statut (ex. bombarder, catapulter, pousser, reconduire, mettre, déceler etc.) - contrairement à ceux qui renvoient à des cérémonies ou rituels officiels spécifiques (ex. couronner, adouber, baptiser etc.). L'ajout du PSR - ou en d'autres mots la CVN - semble donc être responsable de ce changement de sens. Nous avons $\mathrm{vu}$ également que cette construction pouvait faire fi de différentes restrictions généralement imposées par le verbe : en effet, on observe que certains verbes, qui se combinent généralement avec un $\mathrm{SN}$ inanimé peuvent sélectionner, de manière exceptionnelle, un SN animé (ex. détecter, déceler, vérifier, confirmer) et que d'autres, qui requièrent habituellement un certain type de complément comme, par exemple, un syntagme prépositionnel (ex. affecter, muter, classer, inscrire) ou un nom défini (ex. considérer) peuvent désormais apparaître avec un nom nu. Tout ceci semble résulter du pouvoir coercitif de la $\mathrm{CVN}$, qui sera examiné plus en détail dans la section 5 .

\section{Les phénomènes de coercition comme preuve de l'existence d'une CVN}

Dans cette section, nous allons démontrer que l'ensemble des phénomènes sémantiques et syntaxiques que nous avons observés dans la Section 4 (entre autres) 
peuvent être facilement expliqués si l'on postule que la CVN est une construction dans le sens de la Grammaire des Constructions (cf. Goldberg 1995), c'est-à-dire qu'elle constitue une paire forme-sens (" form-meaning pairing ») indépendante des verbes lexicaux qui y entrent. Ainsi, nous proposons de représenter la CVN de la manière suivante:

\begin{tabular}{|lc|}
\hline Syntaxe : & SN1 V SN2 $\mathrm{X}_{\mathrm{Z}}$ \\
Sémantique : & «SN2 acquiert le statut $\mathrm{Z}$ au \\
& $\begin{array}{c}\| \text { terme du procès dénoté par } \mathrm{V} » \\
\end{array}$
\end{tabular}

Schéma 1. La Construction à Verbes de Nomination (=CVN) en Français.

D'après le schéma 1, la CVN est donc caractérisée par la forme syntaxique « SN1 V $\mathrm{SN} 2 \mathrm{X}_{\mathrm{Z}}$ » qui est associée au sens « SN2 acquiert le statut $\mathrm{Z}$ au terme du procès dénoté par V ». Comme nous l'avons déjà mentionné dans la Section 1, nous supposons que cette construction est une instance d'une construction plus schématique, i.e. la CRA (cf. Goldberg 1995, Goldberg \& Jackendoff 2004).

Dans la section précédente, nous avons vu que la CVN avait la capacité de faire rentrer un certain nombre d'items lexicaux dans la position verbale, et ceci indépendamment des restrictions qui pèsent sur ces derniers. Nous avons alors fait référence à la notion de « coercition » (cf. Lauwers \& Willems 2011) : la coercition renvoie - comme son nom l'indique - à tout type de phénomènes dans lesquels des éléments sont insérés " de force » dans une construction dans laquelle ils ne devraient a priori pas apparaitre. En ce qui concerne la $\mathrm{CVN}$, nous avons vu que cette dernière pouvait coercer :

(i) le sens lexical du verbe

Certains verbes ne peuvent être interprétés comme dénotant un procès de nomination que lorsqu'ils sont employés au sens figuré : c'est le cas par exemple de bombarder, catapulter, parachuter, balancer et pousser (cf. Section 4.2.2) et d'estampiller et de tamponner (cf. Section 4.2.7). Or ce sens figuré n'est disponible que lorsque ces verbes sont insérés dans la CVN. En effet, lorsque l'on omet le PSR, comme c'est le cas dans les exemples suivants, les verbes mentionnés ci-avant reprennent un sens littéral et ne peuvent plus être interprétés comme verbes de nomination :

(58) Dali Benssalah a été bombardé/catapulté/balancé/poussé *(tête d'affiche).

(59) Ce designer a été estampillé *("spécialiste du beau").

(60) Ce président a été tamponné *(conforme) par le FMI.

(ii) Les restrictions de sélection du verbe

Comme déjà mentionné dans la Section 4, certains verbes peuvent sélectionner un SN animé seulement lorsqu'ils apparaissent dans la CVN. Pour rappel, c'est le cas des 
verbes comme détecter et déceler (cf. (61)) mais aussi de confirmer (cf. (62)) qui requièrent en temps normal un $\mathrm{SN}$ inanimé. N'oublions pas de mentionner qu'à l'inverse, d'autres verbes, tels que couronner et sacrer (cf. Section 4.2.1) peuvent sélectionner un SN inanimé, à condition qu'ils se combinent à un PSR. Comme montré dans l'exemple (63), l'ajout de cet élément permet à ces verbes de prendre un sens figuré et de s'ouvrir par la même occasion à d'autres types de SNs :

(61) Thierry a été décelé/détecté *(séropositif)

(62) Gérard Linard a été confirmé*(président fédéral de l'union belge de football)

(63) Fais pas ci, fais pas ça a été couronné/sacré *(meilleure série)!

(64) Le 5 juillet 1962, le pays a été déclaré/proclamé *(indépendant).

Il ne s'agit pas toujours d'un contraste entre SNs animés et inanimés. En effet, certains verbes comme, par exemple, déclarer et proclamer (cf. Section 4.2.4) semblent accepter des types de SNs inanimés différents selon qu'ils apparaissent dans la CVN ou non. Ainsi, on ne peut pas déclarer ou proclamer un pays (cf. (64)), mais on peut déclarer la guerre/paix et proclamer l'état d'urgence (cf. la notion de « classes d'objets » de Gross 2008). C'est donc seulement dans le premier cas que le PSR est obligatoire. Notez que l'exemple (64) contraste avec les exemples suivants, dans lesquels les verbes acclamer et saluer - qui décrivent également des actes de communication - apparaissent avec un PSR optionnel :

(65) Vespasien est salué (empereur) par les légions d'Orient

(66) Jon Snow est acclamé (roi du Nord)

(iii) La catégorie morphosyntaxique du complément du verbe

Nous avons vu que la CVN pouvait également avoir un impact sur la catégorie morphosyntaxique du complément de certains verbes, comme par exemple affecter et muter (cf. Section 4.2.3), classer et inscrire (cf. Section 4.2.6) mais aussi se hisser (cf. Section 4.2.2). En effet, en temps normal, tous ces verbes prennent comme complément verbal un SP (ex. affecter/muter quelqu'un à la direction, se hisser à la tête du parti, classer un produit dans la catégorie des substances cancérogènes, inscrire un édifice sur/à la liste des monuments historiques). Pourtant, lorsqu'ils apparaissent dans la CVN, ces verbes peuvent également prendre un nom nu, comme illustré dans les exemples (21), (33), (34), (52) et (54). Nous avions fait la même observation concernant le verbe considérer, qui a fortement tendance à se combiner à un nom défini ou indéfini introduit par comme (ex. je le considère comme le diable en personne/un moins que rien) lorsqu'il est inséré dans la construction dépictive mais qui s'avère être attesté avec un nom nu (cf. monument historique) lorsqu'il est inséré dans la CVN, comme illustré dans l'exemple (7) et répété ci-dessous :

(67) Considéré monument historique par la législation des Monuments Historiques établie en 1914, il est inscrit au patrimoine mondial de l'Unesco en 1985. 
Supposer qu'il existe une CVN, telle que représentée sur le Schéma 1, nous permet de rendre compte de l'ensemble des phénomènes de coercition observés jusqu'ici : ainsi concernant les cas illustrés en (i) (ex. bombarder X tête d'affiche) et une partie des cas illustrés en (ii) (ex. couronner $X$ meilleure série), il semble que le verbe soit imprégné du sens de la CVN tout en gardant son sens de base - lui permettant ainsi de prendre un sens métaphorique qui n'est pas disponible en dehors de la construction, i.e. en l'absence du PSR (cf. (58)) ou dans une autre construction contenant le même PSR (ex. *son bombardement au poste de ministre vs. sa promotion au poste de ministre). Concernant l'autre partie des cas illustrés en (ii) on peut supposer que les verbes déceler et détecter sont attirés par la CVN (en dépit de leurs critères de sélection) par l'intermédiaire d'un réseau constructionnel puisque ces deux verbes apparaissent dans la même construction transitive à SN inanimé que diagnostiquer (ex. diagnostiquer/déceler/détecter une maladie) - qui est lui-même attesté dans la construction transitive à SN animé (ex. diagnostiquer un patient) et la CVN. En d'autres termes, partageant la même construction transitive à SN inanimé, ces verbes viennent à apparaître aussi dans la $\mathrm{CVN}$ par extension analogique. Enfin, pour les cas illustrés en (iii) c'est encore la construction qui légitime l'emploi du nom nu puisque c'est la catégorie morphosyntaxique par défaut pour exprimer un nom de statut (aussi bien dans les phrases copules que dans la CVN).

\section{Conclusion}

Alors que la CRA est généralement décrite dans la littérature comme étant peu productive dans les langues romanes - dont le français -, ceci ne semble pas être le cas de la CVN, qui appartient pourtant à la même famille de constructions. En effet, comme nous l'avons démontré dans cet article, cette dernière s'avère disposer d'une grande ouverture lexicale du point de vue de la position verbale, que l'on peut attribuer à sa capacité à « coercer » certains verbes de sorte à ce qu'ils s'y insèrent. Les effets de coercition peuvent d'ailleurs s'observer à différents niveaux, i.e. au niveau (i) du sens lexical du verbe, (ii) de ses restrictions sélectionnelles, (iii) de la catégorie morphosyntaxique de son complément et, enfin, (iv) de sa structure argumentale. 
7. Annexe.

\begin{tabular}{|c|c|}
\hline Semantic verb class & Number of verbs \\
\hline Crown verbs & $\begin{array}{l}\text { adouber, anoblir, armer, assermenter, baptiser, } \\
\text { béatifier, canoniser, césariser, consacrer, } \\
\text { constituer, (se) convertir, couronner, décorer, } \\
\text { distinguer, établir, honorer, inaugurer, } \\
\text { impatroniser, installer, instaurer, instituer, } \\
\text { introduire, introniser, investir, médailler, } \\
\text { nationaliser, naturaliser, officialiser, oindre, } \\
\text { ordonner, oscariser, primer, sacraliser, sacrer, } \\
\text { sanctifier, titrer, titulariser, récompenser, (se) } \\
\text { reconvertir (se) recycler }\end{array}$ \\
\hline Promote verbs & $\begin{array}{l}\text { balancer, bombarder, catapulter, caser, } \\
\text { grimper, élever, (s')ériger, (se) hisser, } \\
\text { parachuter, passer, pistonner, placer, } \\
\text { positionner, précipiter, projeter, promouvoir, } \\
\text { propulser, mettre, monter, reléguer, rétrograder }\end{array}$ \\
\hline Designate verbs & $\begin{array}{l}\text { admettre, affecter, choisir, coopter, déléguer, } \\
\text { désigner, élire, embaucher, mandater, muter, } \\
\text { nommer, plébisciter, reconduire, recruter, } \\
\text { sélectionner }\end{array}$ \\
\hline Declare verbs & $\begin{array}{l}\text { acclamer, annoncer, s'avouer, déclarer, } \\
\text { décréter, ovationner, proclamer, prononcer, } \\
\text { rapporter, signaler, saluer }\end{array}$ \\
\hline Confirm verbs & $\begin{array}{l}\text { accréditer, agréer, approuver, authentifier, } \\
\text { breveter, certifier, confirmer, entériner, } \\
\text { garantir, habiliter, légaliser, légitimer, ratifier, } \\
\text { valider }\end{array}$ \\
\hline Diagnose verbs & $\begin{array}{l}\text { contrôler, déceler, découvrir, démasquer, } \\
\text { dépister, détecter, déterminer, diagnostiquer, } \\
\text { donner, évaluer, identifier, juger, noter, prouver, } \\
\text { reconnaître, (se) révéler, scanner, tester, vérifier }\end{array}$ \\
\hline Classify verbs & $\begin{array}{l}\text { cataloguer, catégoriser, classer, classifier, } \\
\text { compter, considérer, déclasser, enregistrer, } \\
\text { estampiller, étiqueter, ficher, inscrire, labelliser, } \\
\text { qualifier, répertorier, tamponner }\end{array}$ \\
\hline Residual verb class & faire, imposer, s'improviser, porter \\
\hline
\end{tabular}

Tableau 2. Les verbes attestés dans la CVN répartis selon les 7 classes sémantiques. 


\section{Références bibliographiques}

Aarts, Bas. 1992. Small Clauses in English: The Non-Verbal Types. Topics in English Linguistics 8. Berlin New York: M. de Gruyter.

Boas, Hans Christian. 2003. A Constructional Approach to Resultatives. Stanford Monographs in Linguistics. Stanford, Calif: CSLI Publications.

Chomsky, Noam. 1981. “A Note on Non-Control PRO.” Journal of Linguistic Research $1(4): 1-11$.

- 1986. Barriers. Vol. 13. MIT press.

Dagnac, Anne. 2009. "Elle a Teint Ses Rideaux En Rouge: Entre Manière et Résultativité." Langages, no. 3: 67-84.

Goldberg, Adele E. 1995. Constructions: A Construction Grammar Approach to Argument Structure. Cognitive Theory of Language and Culture. Chicago: University of Chicago Press.

Goldberg, Adele E., and Ray Jackendoff. 2004. "The English Resultative as a Family of Constructions." Language, 532-568.

Guimier, Emilie. 1999. "Les Constructions a Predicat de l'objet En Francais Aspects Syntaxiques, Interprétatifs et Formels."

Gaston Gross. 2008. "Les classes d'objets". Lalies (Paris), Paris: Presses de l'Ecole normale supérieure, pp. 111-165. halshs-00410784

Halliday, Michael. A. K. 1967. Notes on transitivity and theme in English Part I. Journal of linguistics, 3(1), 37-81.

Hoekstra, Teun. 1988. "Small Clause Results." Lingua 74 (2-3): 101-139.

- 1992. "Small Clause Theory." Belgian Journal of Linguistics 7 (1): 125-151.

Hornstein, Norbert, and David Lightfoot. 1987. "Predication and Pro." Language 63 (1): 23.

Lauwers, Peter. 2007. "Nous Sommes ø Linguistes. Quelques Nouvelles Pièces à Verser à Un Vieux Dossier." Neuphilologische Mitteilungen 108 (1): 247-83.

Lauwers, Peter, and Dominique Willems. 2011. "Coercion: Definition and Challenges, Current Approaches, and New Trends." Linguistics 49 (6).

Legendre, Géraldine. 1997. "Secondary Predication and Functional Projections in French." Natural Language \& Linguistic Theory 15 (1): 43-87.

Métairy, Justine, Peter Lauwers, Renata Enghels, Miriam Taverniers, and Marleen Van Peteghem. (2020). "A Micro-Typological Perspective on Resultative Secondary Predicates: The Case of Nomination Verb Constructions." LANGUAGE SCIENCES.

Muller, Claude. 2000. "Les constructions à adjectif attribut de l'objet, entre prédication seconde et complémentation verbale." Langue française 127 (1): 21-35.

Pierrard, Michel. 2001. "Paramètres pour l'interprétation et le classement des constructions à attribut de l'objet." in H. Kronning, C. Norén, B. Novén, G. Ransbo, L.-G. Sundell \& B. Svane (eds), Langage et référence. Mélanges offerts à Kerstin Jonasson à l'occasion de ses soixante ans, Uppsala, Acta Universitatis Upsaliensis : 497-507.

Riegel, Martin. 1991. "Pour ou contre la notion grammaticale d'attribut de l'objet : critères et arguments", in M.-M. De Gaulmyn \& S. Remi-Giraud (eds), À la recherche de l'attribut. Lyon, Presses Universitaires de Lyon : 99-118.

1996. "Les Constructions à Élargissement Attributif: Double Prédication et Prédicats Complexes?" Dépendance et Intégration Syntaxique: Subordination, Coordination, Connexion 351: 189.

- 2001. "Quelques remarques sur les constructions à attribut de l'objet issues d'une complétive attributive. » in H. Kronning, C. Norén, B. Novén, G. Ransbo, L.G. Sundell \& B. Svane (eds), Langage et référence. Mélanges offerts à Kerstin Jonasson à l'occasion de ses soixante ans, Uppsala, Acta Universitatis Upsaliensis : 543-553. 
Talmy, Leonard. 1985. "Lexicalization Patterns: Semantic Structure in Lexical Forms." Language Typology and Syntactic Description 3 (99): 36-149.

_. 1991. "Path to Realization: A Typology of Event Conflation." In Annual Meeting of the Berkeley Linguistics Society, 17:480-519.

Tobback, Els. 2005. "Les Constructions à Attribut de l'objet et Le Marquage de La Relation Prédicative Seconde." Thèse de doctorat, Université de Gand.

Tobback, Els, and Bart Defrancq. 2008. "Un comme qui marque une fonction qui se démarque. L'attribut de l'objet en comme et les verbes de nomination." Langue française 159 (3): 116.

Stowell, Timothy Angus. 1981. "Origins of Phrase Structure.” Massachusetts Institute of Technology.

Van Peteghem, Marleen. 1993. La Détermination de l'attribut Nominal: Étude Comparative de Quatre Langues Romanes (Français, Espagnol, Italien, Roumain). Brepols.

Willems, Dominique. 1981. "Syntaxe, Lexique et Sémantique: Les Constructions Verbales."

${ }^{\mathrm{i}}$ Les exemples cités dans cet article sont tous tirés d'internet (i.e. articles de journal, blogs, forums etc.) sauf mention contraire.

ii Ces constructions résultatives sont dites "analytiques" dans la mesure où le sens résultatif est obtenu par l'intermédiaire d'un constituant à part. Ce sens résultatif n'est donc pas incorporé dans la racine verbale (ex. couper en dés en français vs. dice en anglais)

iii Ce contraste entre les langues germaniques et les langues romanes est attribué à une différences typologique fondamentale observé par Talmy $(1985,1991)$ : alors que les langues germaniques sont des langues à cadrage satellitaire, les langues romanes sont des langues à cadrage verbale. Pour rappel, dans les langues à cadrage verbale, la 'trajectoire' (en anglais, path) d'un évènement (i.e. la direction ou le résultat) a tendance à être incorporé dans la racine verbale (ex. entrer dans la chambre (en dansant), aplatir à coups de marteau). En revanche, dans les langues à cadrage satellitaire, celle-ci est exprimée par l'intermédiaire d'un constituent à part, en dehors du verbe (ex. dance INTO the room, hammer FLAT).

${ }^{\text {iv }}$ Pour une discussion approfondie des propriétés sémantiques et syntaxiques du nom nu dans les emplois prédicatifs en français, voir Van Peteghem (1993) et Lauwers (2007), entre autres.

v L'adjectif est la catégorie morphosyntaxique par défaut dans les CRAs de l'anglais (ex. paint the door red, shoot him dead, water the plants flat).

vi Tobback \& Defrancq (2008) sont, à notre connaissance, les seuls à avoir employé le terme de « construction » à verbes de nomination, sur la base du fait qu'il y ait « de bonnes raisons de croire que la structure elle-même véhicule un sens résultatif, étant donné qu'elle peut « coercer » une lecture résultative de verbes qui n'ont pas ce sens, tels que bombarder $»(\mathrm{p} .120)$

vii La productivité renvoie grosso-modo au domaine d'application d'une construction. La CVN est alors productive dans la mesure où elle s'applique - comme nous le verrons dans la section 4 - à un grand nombre de verbes appartenant à différentes classes sémantiques ainsi qu'à plusieurs catégories morphosyntaxiques lorsqu'il s'agit du PSR (cf. Métairy et al. (2020)). 
viii Parmi ces 140 verbes, 4 ne figurent pas sur ce tableau - il s'agit des verbes faire, imposer, s'improviser et porter (voir Tableau 2 en annexe).

${ }^{\text {ix }}$ Cette alternance syntaxique justifie pour certains une analyse à « petite proposition » (en anglais Small Clause analysis, cf. Stowell 1981; Chomsky 1981, 1986a; Hornstein \& Lightfoot 1987, Hoekstra 1988, 1992; Aarts 1992, voir aussi Legendre 1997 pour le français) selon laquelle le PSR et son sujet - autrement dit la base de la prédication - forme un constituant qui équivaut à une proposition entière sur le plan sémantique. 